\title{
Rede de Cooperação Digital: utilizando o Amadis como recurso metodológico para o desenvolvimento de comunidades de baixa renda
}

\author{
Cristina Schwarz* \\ Marta Dieterich Voelcker** \\ Melissa Meier*** \\ Sabrina Silveira $* * * *$ \\ Susana Seidel $* * * * *$
}

Resumo: As Tecnologias da Informação e Comunicação (TICs) têm trazido muitos benefícios para as comunidades de baixa renda por meio da ação de Organizações NãoGovernamentais (ONGs), especialmente pela possibilidade de utilização de um ambientes de aprendizagem a distância, como o AMADIS. O surgimento da Fundação Pensamento Digital em Porto Alegre se deu pela motivação da sociedade em apoiar ONGs na sua estrutura como um todo. Desse movimento surgiu a Rede de Cooperação Digital, que veio suprir muitas necessidades, desde a infra-estrutura para a tecnologia, até a formação técnico-pedagógica das equipes, principalmente dos educadores das ONGs. O AMADIS está gerando um fascínio por parte dos atores envolvidos, com isso proporcionando interação e cooperação entre as ONGs, melhorando muito o desenvolvimento das atividades já praticadas antes, mas agora com muito mais motivação e autonomia. Serão abordadas, neste relato, as estratégias metodológicas e resultados obtidos com o uso do AMADIS, destacando o vínculo afetivo e social entre as ONGs e a equipe pedagógica da Fundação Pensamento Digital.

Palavras - chave: comunidade virtual, ambiente virtual de aprendizagem.

Title: Digital Cooperation Network: employing Amadis as a methodological resource for the development of low-income communities.

Abstract: The Information and Communication Technologies (ICTs) bring about many benefits to low-income communities through actions of Non-Governamental Organizations (NGOs), particularly due to the possibility of using long distance learning environments such as AMADIS. The creation of Fundação Pensamento Digital (Digital Thought Foundation) in Porto Alegre was a result of society's motivation to support NGOs in its structure as a whole. From this movement arose the Digital Cooperation Network, which turned out to meet many needs that go from infrastructure for technology to technical-pedagogical qualification, specially the educator's. AMADIS is causing allure among the actors involved and hence promoting interaction and cooperation among NGOs - and considerably improving the development of activities that now seem to be held with more motivation and autonomy. This report will present the methodological strategies and result obtained from the employment of AMADIS, emphasizing the affective and social bond between NGOs and the Foundation's team.

Key - words: virtual community, virtual learning environment. 


\section{Introdução}

Neste relato será apresentado uma experiência com o Projeto Rede de Cooperação Digital, criado pela Fundação Pensamento Digital, no intuito de promover a interação e cooperação entre comunidades de baixa renda (Organizações nãoGovernamentais - ONGs) para o desenvolvimento de suas ações, utilizando as tecnologias da informação e comunicação (TICs). É um Projeto dedicado a provisão de computadores reutilizados para ONGs e a criação e suporte técnico-pedagógico de uma comunidade virtual, protagonizada por educadores e aprendizes das ONGs beneficiadas.

O Projeto oferece formação para as equipes destas ONGs visando:

- apropriação da Tecnologia para Cooperação na Internet;

- construção e registro de conhecimento local.

Para isso, utilizou-se como recurso metodológico o ambiente de aprendizagem a distância Amadis. Este ambiente foi criado para ser utilizado em escolas, mas foi adaptado, criando estratégias de uso de seus recursos para a utilização nas ONGs.

Acredita-se que a rede criada entre os diversos educadores e aprendizes cadastrados no Ambiente, irá se construir uma comunidade virtual na internet. Entre os objetivos esperados, listamos: a criação de laços sociais, afetividade, aumento da autoestima e principalmente autonomia para construção e desenvolvimentos de suas ações.

\section{Fundação Pensamento Digital}

Originada em 1999, a partir da mobilização de voluntários pela inclusão digital, formalizada em 2000, a Fundação Pensamento Digital ${ }^{1}$ mobiliza diversos segmentos da sociedade gaúcha para a promoção de projetos educacionais, através do uso das novas TICs. A atuação das universidades como parceiras na orientação pedagógica dos trabalhos, permitiu à Fundação especializar-se na formação de educadores comunitários, para o uso das TICs na promoção do desenvolvimento de comunidades de baixa renda.

Oportunizar a comunidades de baixa renda o acesso a Tecnologia da Informação e Comunicação (TIC), e também promover o uso das TICs para o desenvolvimento sócio-econômico das comunidades beneficiadas, são os principais objetivos da Fundação Pensamento Digital.

A Fundação Pensamento Digital estabelece parceria com empresas, universidades, ONGs e governos para captar, recuperar e distribuir computadores.

\subsection{Projeto Rede de Cooperação Digital}

O Projeto acontece através do ambiente de aprendizagem a distancia onde usuários das diversas ONGs conversam entre si, com voluntários orientadores e equipe pedagógica da Fundação Pensamento Digital; compartilhando idéias e projetos; construindo conhecimento, criando-se assim uma comunidade virtual de aprendizagem.

É oferecido formação para as equipes das ONGs, visando a apropriação da Tecnologia para Cooperação na Internet, construção e registro de conhecimento local.

$\mathrm{O}$ uso das TICs para empoderamento dos diversos projetos sociais em andamentos nas ONGs parceiras, não mais voltadas apenas para cursos de informática, mas para e exploração da aprendizagem em rede, é o principal foco do Projeto. Para o uso das TICs propõe-se a pedagogia de Projetos de Aprendizagem, onde o próprio aprendiz escolhe os problemas a serem pesquisados. 
O método de aprendizagem por projetos tem como "roda motriz" a motivação do aprendiz em resolver um problema ou investigar uma questão escolhida por ele mesmo, permitindo que ele desenvolva formas de trabalho autônoma na construção de seu próprio conhecimento.

A Pedagogia de Projetos de Aprendizagem integrada a Comunidade Virtual de Aprendizagem foi proposta por pesquisadores do Laboratório de Estudos cognitivos da UFRGS.

\section{Tecnologias da Informação e Comunicação (TIC's) no Desenvolvimento das Comunidades}

Com referência a Weigel e Waldburger (2004), partiu-se do pressuposto de que as transformações que ocorrem com a chegada das novas TICs trazem consigo uma possibilidade para a criação de novos caminhos para a redução da pobreza. As TICs têm $\mathrm{o}$ potencial de revolucionar as formas em que habilidades, conhecimento $\mathrm{e}$ competências são adquiridas por indivíduos, organizações e comunidades.

Nesse contexto, certas instituições internacionais, governos e ONGs têm desenvolvido projetos para promover o uso das novas TICs em comunidades de baixa renda. Entende-se que, para isso, esses projetos devem ser planejados, implementados e monitorados com foco em aprendizagem e desenvolvimento de competências.

"O educador não deve pensar apenas na área cognitiva, em um ambiente de aprendizagem construtivista é preciso ativar mais do que o intelecto. Ao educador cabe a função de ativação da aprendizagem, ele deve trabalhar consigo mesmo a percepção de seu próprio valor e promover a auto-estima e a alegria de conviver e cooperar, bem como desenvolver um clima de respeito e de auto-respeito."(FAGUNDES, MAÇADA e SATO, 1999)

Acredita-se que as tecnologias da informação e comunicação podem ajudar essas comunidades a se desenvolverem e trocarem informações entre si. Inclusão digital é mais que permitir o livre acesso a computadores: pretendemos que esses usuários possam fazer parte desse mundo virtual e que possam utilizar essas tecnologias para modificar suas realidades.

\section{Ambiente de Aprendizagem a Distância: Amadis}

$\checkmark \quad$ O ambiente escolhido para o Projeto Rede de Cooperação Digital foi o Amadis (Ambiente de Aprendizagem a Distância). Este ambiente possui recursos que possibilitam as interações planejadas no Projeto. Além disso, a utilização de um ambiente está apoiada na teoria de Piaget, como diz Nevado (2001): Um ambiente de aprendizagem constitui-se em um convite para aprender. Piaget afirma que as crianças e também os adultos deveriam ter oportunidades para realizar suas próprias experimentações e suas próprias pesquisas. Tais explorações e experimentações implicam em uma certa ludicidade, um interjogo dos recursos internos do sujeito (tanto afetivos quanto cognitivos, estéticos, éticos, etc.) com os objetos do ambiente e as interações com outros sujeitos.

O Amadis foi desenvolvido apoiado por um convênio entre UFRGS, a Prefeitura de Porto Alegre e o BNDES, dentro da linha Programática Atenção Integral ao Adolescente e ao Jovem. A partir do uso, a equipe de desenvolvimento notou algumas 
mudanças importantes e necessárias a serem feitas. Com isso, atualmente utiliza-se uma nova versão do ambiente, com uma interface mais atraente e recursos de comunicação mais desenvolvidos. Será apresentado nesse relato tanto as atividades realizadas na versão anterior quanto na atual.

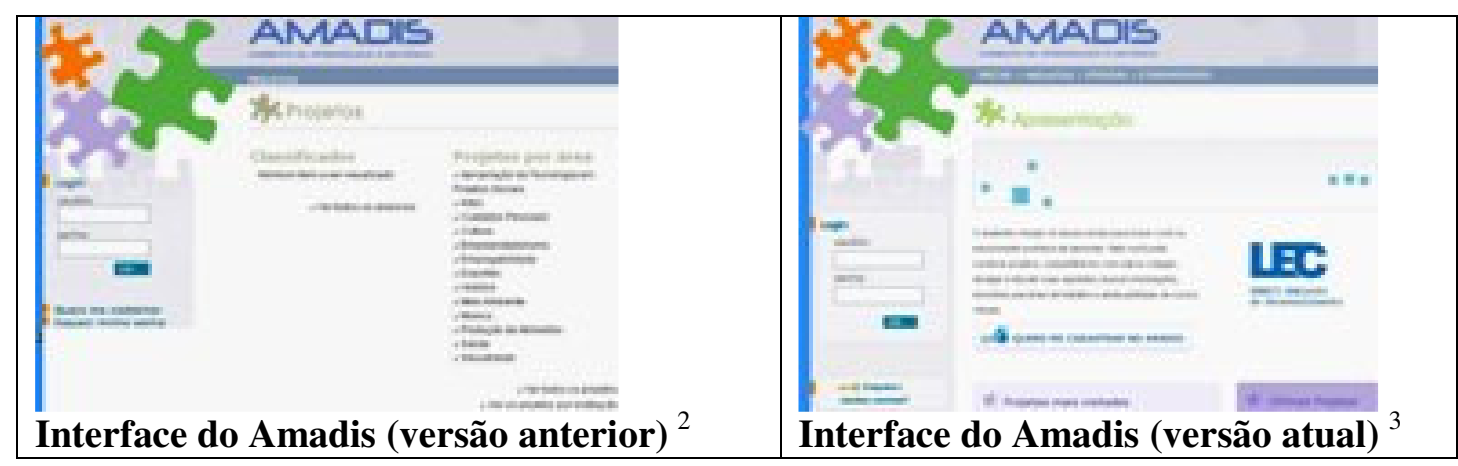

\section{Estratégias Metodológicas e Resultados Obtidos}

As estratégias metodológicas de uso de cada um dos recursos do Amadis, planejadas e executadas pela equipe da Fundação Pensamento Digital serão apresentadas a seguir.

\subsection{Páginas Pessoais}

Neste recurso os usuários inscritos no Ambiente têm a possibilidade de publicarem páginas HTML em um espaço reservado para upload de arquivos pessoais. Este recurso tem sido utilizado com dois objetivos: aprendizado de recursos tecnológicos e a construção de uma identidade virtual dentro do Ambiente.

No primeiro objetivo, foi solicitado que os usuários criassem uma página pessoal, utilizando o Mozilla Composer ${ }^{4}$. Buscou-se, com esse recurso, despertar nos usuários a curiosidade e o desejo por novos conhecimentos tecnológicos. Para isso foi utilizado como estratégia a apresentação de páginas já publicadas por outros usuários, que acabaram se tornando referenciais para os novos, e a disponibilização de materiais de apoio 5 .

No segundo objetivo, a página pessoal é apresentada como um "cartão de visitas", onde o usuário tem a possibilidade e a liberdade de se apresentar, mas agora dentro de uma realidade virtual ${ }^{6}$.

A página do Deivis e a da Fabiola são exemplos de utilização dos recursos tecnológicos trabalhados na formação oferecida pela Fundação Pensamento Digital, além de serem exemplos do trabalho realizado com a auto-estima dos sujeitos:

Deivis - http://amadis_fpd.lec.ufrgs.br/paginas/users/user_27/

Fabiola - http://amadis_fpd.lec.ufrgs.br/paginas/users/user_13/fabiola.html

\subsection{Diário}


Neste recurso o usuário dispõe de um local individual para postar suas reflexões, ficando estas visíveis e disponíveis para comentários a todos os cadastrados no ambiente.

Utilizou-se como estratégia comentar estes diários de forma a interagir mais diretamente com os usuários, buscando criar vínculos, esclarecendo dúvidas, instigando novos conhecimentos, apoiando e incentivando trabalhos.

08/11/2005 - Faltando 10 dias para completar um ano que a equipe da pensamento digital esteve na ong Maria Mulher para dar ínicio ao projeto, hoje finalmente estamos com ele em andamento agora já conectados pode no dia de hoje mais técnicas da ong participando do processo confesso que estou muito feliz pois posso dizer que eu fui umas das pioneiras a conhecer este projeto e ver que ele realmente funciona e que podemos utiliza-lo é realmente muito gratificante. Neste primeiro dia de capacitaçăo estiveram presente Décio e Susana pela Fundaçăo e integrantes da equipe de Maria Mulher, Eu Gilciane,Eliana, Caroline, Dolores e Rosélia, poucas mas muitas para um pequeno ínicio, estavamos todas entusiasmadas com essa novidade e confesso que fiquei com a impressăo de que esse projeto realmente poderá fazer a diferença.

\section{Diário da Gilciane no Amadis}

Você é uma das pessoas que mais acreditam no projeto e que batalham para que as coisas aconteçam! Parabéns pelo teu esforço para conseguir a internet para a ONG. O potencial do projeto é incrivel, ainda mais quando pessoas interessadas estăo envolvidas... Vocẻ fol uma das pioneiras e é motivo de orgulho para toda a equipe! Conseguimos passar a idéia do projeto para vocé.

Espero que agora possamos trabalhar com as tuas colegas da ONG e acredito que com a sua ajuda e vontade elas logo estarăo engajadas no nosso projeto, utilizando a informática para promover o aprendizado das suas próprias alunas, nas oficinas de cada uma. Ahhh! Vamos nos comunicar com maior frequểncia pelo diário, ok?

Beijos

\section{Comentário da equipe da Fundação Pensamento Digital}

Acima foi exemplificado um reconhecimento e estímulo ao usuário por parte da equipe da Fundação.

Este mesmo diário $(08 / 11 / 2005)$ foi comentado por uma usuária de outra instituição, com uma solicitação de ajuda. Mesmo não se conhecendo, o ambiente de confiança criado no Amadis, permitiu que a "usuárial" se apresentasse e construísse (nas interações que aconteceram) laços sociais com a Gilciane.

$$
\text { 22/17/2005 ol,Gil como vai?Meu nome é }
$$

aluna do Décio moro aqui no campo da tuca.Estou passando por um momento dificil da minha vida meus pais moram longe.Moro com meus tres filhotes e tambem faço niver em maio(26). Bom resolvi me separar mas ele nắo aceitou como eu esperava,já faz 5 meses qu foi morar com a mắe dele mas no ultimo dia(12) me agrediu ciuma faca na minha casa.Espero qf vc possa me ajudar mas desde já adorei ter te conhecido.Beijos

Comentário da "usuárial” no diário da Gilciane

Outra estratégia adotada foi a utilização dos diários da equipe pedagógica da Fundação Pensamento Digital, como um local disponível para questionamentos e trocas. Assim, os usuários têm a possibilidade de interagir comentando o diário da equipe da 
Fundação com o qual criou um vinculo afetivo, estando assim mais confortável para fazê-lo. Veja um exemplo desta estratégia, abaixo:

\section{Atividades no Amadis - 02:38 11/05/2006}

Tenho lido as construções do pessoal do curso básico (24/04). Eles estão refletindo bastante sobre o uso de tecnologias e isso me deixa muito satisfeita, por acreditar que contribuimos com eles nesse sentido. Ao mesmo tempo estou sentindo a necessidade de me aprimorar em novos recursos para poder apresentar nos próximos cursos. Por esse motivo vou modificar a minha página pessoal usando recursos mais avançados. Muito trabalho...

Diário de um integrante da equipe da Fundação

Ola como vai as coisas, como vc colocou uma barra de rolagem no meio da sua pagina pessoal... Vim tb dizer que publiquei mais uma pagina, se vo puder de uma olhadinha, algumas fotos nẫo aparecern, pq alguem ak apagou elas, e tb o conteudo ern si é só o inicio. obrigado pela sua atençẫo. (deivis, 18,05/2006)

Comentário feito no diário do integrante da Equipe da Fundação

\subsection{Fórum}

Assim como no recurso do diário, neste recurso procurou-se auxiliar aos usuários em seus questionamentos, mas agora com uma estratégia diferenciada: aguardar os questionamentos ou comentários dos usuários para então auxilia-los.

É essencial haver um local aberto para questionamento onde os usuários tenham a possibilidade tanto de questionar como auxiliar aos demais.

\footnotetext{
OI PESSOAL:"m:m:m (magda nice barrada, 2/3/2005)

ESTOU A MUL COM MINHAS ANOTACODES, MAS ESBARREI MUMA QUESTÃO: ENTRAR NO COMPOSER FOI FACIL MAS AS SENHAS CONTINUAM ME BARRANDO, CONO ESCREVI NA MENSAGEM ANTERIOR TINHAMOS OUAS SENHAS UMA PARA DIFERENTES TAREFAS. UMA PARA COMPOR A PAGINA PESSOAL OUTRA QUANDO MUDAMOS PARA O PROJETO. FUI PROCURAR NWHA PASTA E MABO CONSEGUI ABRI-LA. PARECE QUE NÄO EXISTE, NELA ESTÄO TODAS AS MINHAS ANOTACÖES, FOTOS ETC. QUE POSSO EN AULA MOSTRAR PARA OS ALUNOS, PARA QUE VEJAM COMO FICOU MNHA PASTA ANTES DE MONTAR MNIHA PAGINA. DESDE JÁ AGRADECYO, UM ABRAÇO MAGDA

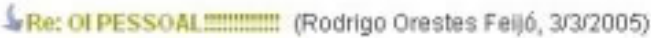

Oi Magda $O$ que acontece é que essas senhas que tu anotaste sao para acessar as computadores do LEC (que são protegidas por senhas). A mudança que houve nas senhas foi para evitar que os computadiores (aqui do [EC) travassem no meio do cursa. Essas senhas näo seräo necessánias para o curso que vacé vai resilizar ai no Rubem Berta (pois os computadores nåo sto protegidos por senhs). Para utilizar o camposer nǔo é necessário senhas, apenas acessar o mozilla. Partanto, a nö̆o ser que venha nos visitar no LEC, essas senhas e usuánios nక̄o serăo mais necessánios. Quanto sas arquivos, você só poderá acessar os arquivas publicadas no AMADIS. Espero ter esclarecido. Abraço. Rodrigo

Ol RODPGGotin:min: (magda nice barrada, 3/3/2005)

OBRIGADA POR RESPONOER, MAS AGORA É QUE A PORCA TORCEU O RABO. ENTREI NO AMADIS, FUI NO PRONETO, NO ARQUIVO DO PROJETO E O QUE EU CONSEGUI FOI APENAS VER MEUS ARQUIVOS SEM AS SENHAS, QUE NẢO VALEM PARA ESTE COMP COMO POSSO ENTRAR NO MEU AROUIVO? QUAL OS CAMNHHOS PARA ISSO? E COMO VOU (POR EXEMPLO),ANEXAR NOVOS DADOS NO MEU PROJETO, AGORA A DISTÁNCIA SE MAZO ENTRO MOS ARQUIVO? POR FAVOR MÄO ME DEIXEN SÖm!!m!!m!m!m!!! UM ABRACO, MAGDA

Fórum "Curso de Páginas no Rubem Berta"
} 
O fórum acima foi criado especificamente para auxiliar técnicapedagogicamente a educadora Magda (aluna da primeira formação oferecida pela Fundação Pensamento Digital) e seus alunos em um curso que esta estava ministrando no Telecentro Rubem Berta.

Acredita-se também, que estes fóruns, com todos os seus questionamentos os quais podem ser também questionamentos de futuros usuários do ambiente, servirão como material de referência, assim como são as páginas pessoais.

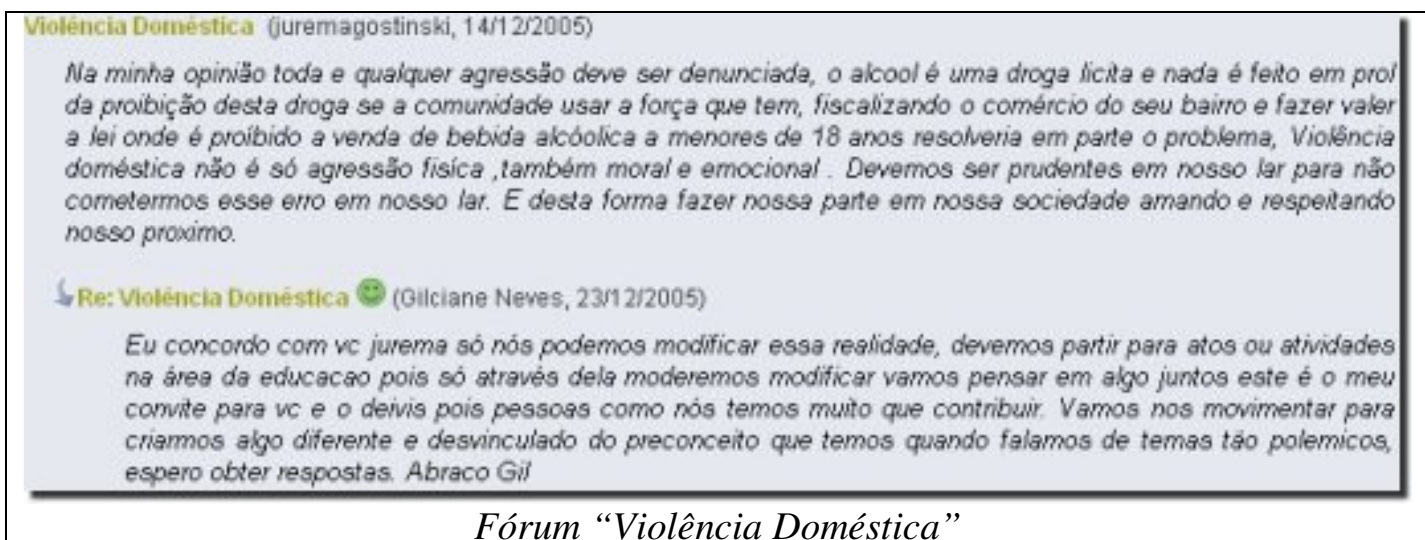

No fórum acima, Gilciane, que trabalha no Maria Mulher ${ }^{6}$, colabora com outra usuária nesse tema que é tão presente em seu trabalho.

\subsection{Chat}

Este recurso é utilizado para comunicação em tempo real com uma previa marcação de datas e horários.

Utilizou-se como estratégia não estabelecer um único tema a ser discutido, mas sim aproveitar as colocações que surgem, durante o chat, para que se faça intervenções que possam gerar reflexões nos sujeitos.

\subsection{Projetos de Aprendizagem}

O objetivo principal do ambiente que está sendo utilizado é auxiliar no desenvolvimento de Projetos de Aprendizagem (PA). O que é PA? Inicia-se um projeto a partir de uma questão principal sobre um assunto. Após, é preciso refletir sobre o que já se sabe sobre esse assunto e o que se quer saber. $\mathrm{O}$ próximo passo é partir para a pesquisa e registrar as descobertas feitas.

"Quando falamos em "aprendizagem por projetos" estamos necessariamente nos referindo à formulação de questões pelo autor do projeto, pelo sujeito que vai construir conhecimento. Partimos do princípio de que o aluno nunca é uma tábula rasa, isto é, partimos do princípio de que ele já pensava antes.” (FAGUNDES, MAÇÂDA e SATO, 1999)

No trabalho com as comunidades uma das estratégias é possibilitar um espaço de divulgação das iniciativas, propostas e projetos das instituições comunitárias, de forma a gerar uma rede de colaboração e cooperação entre elas. Ou seja, muitas vezes, no lugar de publicarem PA's, os usuários publicam as páginas ou projetos de suas comunidades. 
Vejam alguns exemplos de Projetos de Aprendizagem criados no Amadis, através do Projeto Rede de Cooperação Digital:

Transgênicos: http://fpd.lec.ufrgs.br/amadis/paginas/projeto_20/

Doação de Sangue: http://fpd.lec.ufrgs.br/amadis/paginas/projeto_23/

Vejam alguns exemplos de páginas de comunidades e seus projetos, publicadas no Amadis, através do Projeto Rede de Cooperação Digital:

Movimento pelos Direitos das Crianças e Adolescentes:

http://amadis_fpd.lec.ufrgs.br/paginas/projetos/projeto_6/

Página do Núcleo Comunitário de Belém Novo:

http://amadis_fpd.lec.ufrgs.br/paginas/users/user_27/intituisao.html

Inclusão Digital no Centro de Capacitação Profissional Milton Santos: http://amadis fpd.lec.ufrgs.br/paginas/projetos/projeto 1/

\section{Conclusão}

De acordo com o indicador Nacional de Analfabetismo Funcional (Instituto Paulo Montenegro ${ }^{8}, 2005$ ), apenas $26 \%$ da população brasileira tem domínio pleno das habilidades de leitura e escrita. Significa que $74 \%$ da população não entende o que lê e não consegue redigir um texto. No Projeto Rede de Cooperação Digital os resultados obtidos superam as expectativas em relação a esse índice. Os usuários do AMADIS desenvolvem essas habilidades com o auxílio do uso das tecnologias na comunidade virtual gerada pelo ambiente.

A formação dos educadores e demais integrantes das ONGs beneficiadas pela Rede de Cooperação Digital, com base na pedagogia de projetos de aprendizagem, integrada a construção e alimentação da comunidade virtual, mostrou-se apropriado para oportunizar uma série de vivências, que contribuem para que compreendam e se integrem às mudanças em curso na sociedade.

Ao criarem conteúdos e ao participarem de fóruns e chats no AMADIS, os usuários se expressam muito através da escrita. Além disso, demonstram compreensão em relação às informações obtidas em pesquisas em livros e na internet.

$\mathrm{O}$ principal resultado deste relato foi comprovar que é possível, a partir da proposta de formação, utilizando o Amadis como recurso metodológico para o desenvolvimento de comunidades de baixa renda, construir um espaço de inclusão onde os usuários das ONGs possam se pensar como sujeitos de seu próprio processo de viver, construindo seus próprios instrumentos de inclusão.

\section{Notas}

${ }^{1}$ - Site da Fundação Pensamento Digital: http://www.pensamentodigital.org.br

2- Site da versão anterior do Amadis, utilizada pela Fundação Pensamento Digital: http://fpd.lec.ufrgs.br/amadis

3 - Site da versão atual do Amadis, utilizada pela Fundação Pensamento Digital, desde abril de 2006: http://amadis_fpd.lec.ufrgs.br 
4 - Mozilla Composer: software livre que permite a construção de documentos no formato HTML. Site de origem: http://www.mozilla.org/

5 - Sites dos materiais de apoio: http://oficinas.lec.ufrgs.br , http://oficinas.psico.ufrgs.br/html e http://oficinas.lec.ufrgs.br/wiki

${ }^{6}$ - Encaramos como realidade virtual as trocas que são feitas a distância através do ambiente.

7- Site da Organização para Mulheres Negras - Maria Mulher: http://www.portalafro.com.br/entidades/mariamulher.htm

E-mail: mariamulher@cpovo.net

${ }^{8}$ - Instituto de Pesquisa Econômica Aplicada - IPEA. http://www.ipea.gov.br

\section{Referências}

FAGUNDES, L.C. MAÇADA, D.L.; SATO, L.S. Aprendizes do Futuro: as Inovações Começaram. Coleção Informática para a Mudança na Educação Ministério da Educação. Brasília: Estação Palavra, 1999.

NEVADO, R.A.; MAGDALENA, B.C.; COSTA, I.T. Formação de Professores Multiplicadores:nte2@ projetos.cooperativos.ufrgs.br. LEC/UFRGS 2001.

WEIGEL, G., WALDBURGER, D. (editores). ICT4D - Connecting People for a Better World - Lessons, Innovations and Perspectives for Information and Communication Technology in Development. Publicado pela Swiss Agency for Development and Cooperation (SDC) and the Global Knowledge Partnership (GKP). Berna, 2004. 